\title{
MODELLING OF BLOOD'S NON-NEWTONIAN BEHAVIOUR IN PATIENT-SPECIFIC AORTO-CORONARY BYPASS GRAFTS
}

\author{
J. Vimmr ${ }^{1}$, A. Jonášová ${ }^{1}$, O. Bublík ${ }^{1}$ \\ ${ }^{1}$ Department of Mechanics, University of West Bohemia (jvimmr@kme.zcu.cz)
}

\begin{abstract}
The patency and overall performance of implanted bypass grafts is closely related to hemodynamics and its influence on vessel remodelling. In this regard, numerical investigation of blood flow in models reconstructed from clinical data may, next to clinical research, provide a valuable insight into the problem of graft failures, which are usually associated with restenosis and/or occlusive intimal hyperplasia. In this study, numerical results of pulsatile non-Newtonian blood flow in three realistic aorto-coronary bypass models are presented and further discussed with emphasis placed on the distribution of wall shear stress (WSS) and oscillatory shear index (OSI). Blood's shear-thinning behaviour is described by the CarreauYasuda model. Assuming all model walls to be impermeable and inelastic, the numerical solution of the mathematical model, which has the form of time-dependent non-linear system of Navier-Stokes (NS) equations, is carried out on the basis of the three-stage fractional step method and cell-centred finite volume method formulated for hybrid unstructured tetrahedral grids. The viscous terms of the NS equations are time discretised implicitly using the CrankNicolson scheme. The convective terms are solved explicitly and their computation utilises a local time-stepping technique in order to improve the overall computational efficiency of the developed CFD code.
\end{abstract}

Keywords: Aorto-coronary bypass graft, pulsatile blood flow, Carreau-Yasuda model, Finite volume method, Fractional-step method.

\section{INTRODUCTION}

Nowadays it is generally accepted that the performance and patency of implanted bypass grafts may be significantly affected by local hemodynamics, [11]. Beside thrombogenesis, usually originating in low flow rates or technical mistakes, [14], the majority of recorded bypass failures is often caused by intimal hyperplasia, [6]. This type of intimal thickening represents a form of abnormal healing process observed at several sites of the distal anastomosis, Fig. 1. The associated morphological and metabolic changes within the vessel walls are, among others, hypothesised to be triggered by disturbed blood flow and low and oscillating shear stress, [1]. In light of this knowledge, an investigation of hemodynamics in the form of numerical simulations may be a valuable contribution to the understanding of graft disease formation. 


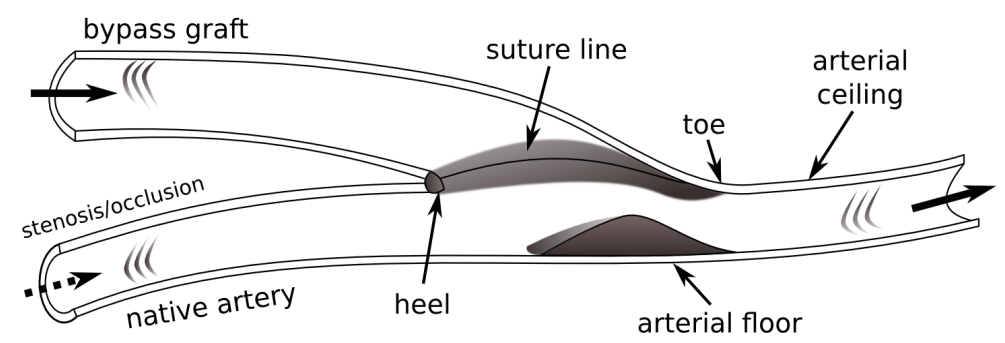

Figure 1. Localisation of intimal thickening at the distal end-to-side anastomosis with relevant terminology, modified from [1].

\section{BYPASS MODELS AND PROBLEM FORMULATION}

Based on our existing experience with steady non-Newtonian blood flow in idealized bypass models, [13], the main objective of the present study is to model bypass hemodynamics in patient-specific aorto-coronary grafts by considering flow pulsatility and blood's nonNewtonian behaviour. For this purpose, we distinguish two types of bypass grafts - individual and sequential ones. In cardiovascular surgery, the term individual graft denotes a bypass with one distal end-to-side anastomosis, i.e., the implanted graft provides a direct connection between the aorta and the stenosed or occluded coronary artery. In the case of the sequential graft, at least two distal anastomoses are present. Namely, the implanted graft supplies blood to several coronary arteries in the form of one or several side-to-side anastomoses.

In this study, three different aorto-coronary bypass models are considered with individual and/or sequential grafts, Fig. 2. In other words, pulsatile blood flow will be modelled in single, double and triple bypasses (in this case, we refer to the number of coronary arteries bypassed in the procedure). All the models, which connect aorta to the branches of the left and/or right coronary artery, are reconstructed from CT and MRI data provided by the courtesy of the University Hospital in Pilsen, Czech Republic. The reconstruction process was carried out in software package Amira (primary reconstruction) and continued in the software Altair Hypermesh (smoothed model), Fig. 2. In the last mentioned software, an unstructured tetrahedral computational mesh for each of the bypass models was generated, Figs. $3-5$. According to a preliminary grid sensitivity analysis, the mesh size for the single, double and triple bypasses was chosen to be $362,437,476,241$ and 879,480 tetrahedral cells, respectively.

The simulation parameters are chosen in accordance with other studies devoted to the modelling of bypass hemodynamics, see the review paper [11]. Firstly, taking into account the fact that at the end of the arterialisation process, venous grafts lose their compliance, all the bypass walls are modelled as impermeable and inelastic, including the wall of the aorta. In the light of this simplification, we are aware that the neglected aortic elasticity represents a considerable limitation of the present study. We hope to rectify it in one of our future projects by solving the fluid-structure interaction problem. In this study, we further assume a static aorto-coronary bypass model, i.e., the influence of heart beating on the resulting blood flow is not considered. This assumption is based on the conclusion drawn in [16], where it was shown that the arterial motion does not significantly affect coronary blood flow when flow pulsatility is considered. In accordance with the studies devoted to the hemodynamics in aorto-coronary bypasses, $[17,5,8]$, we further assume the blood flow to be pulsatile and laminar, neglecting 


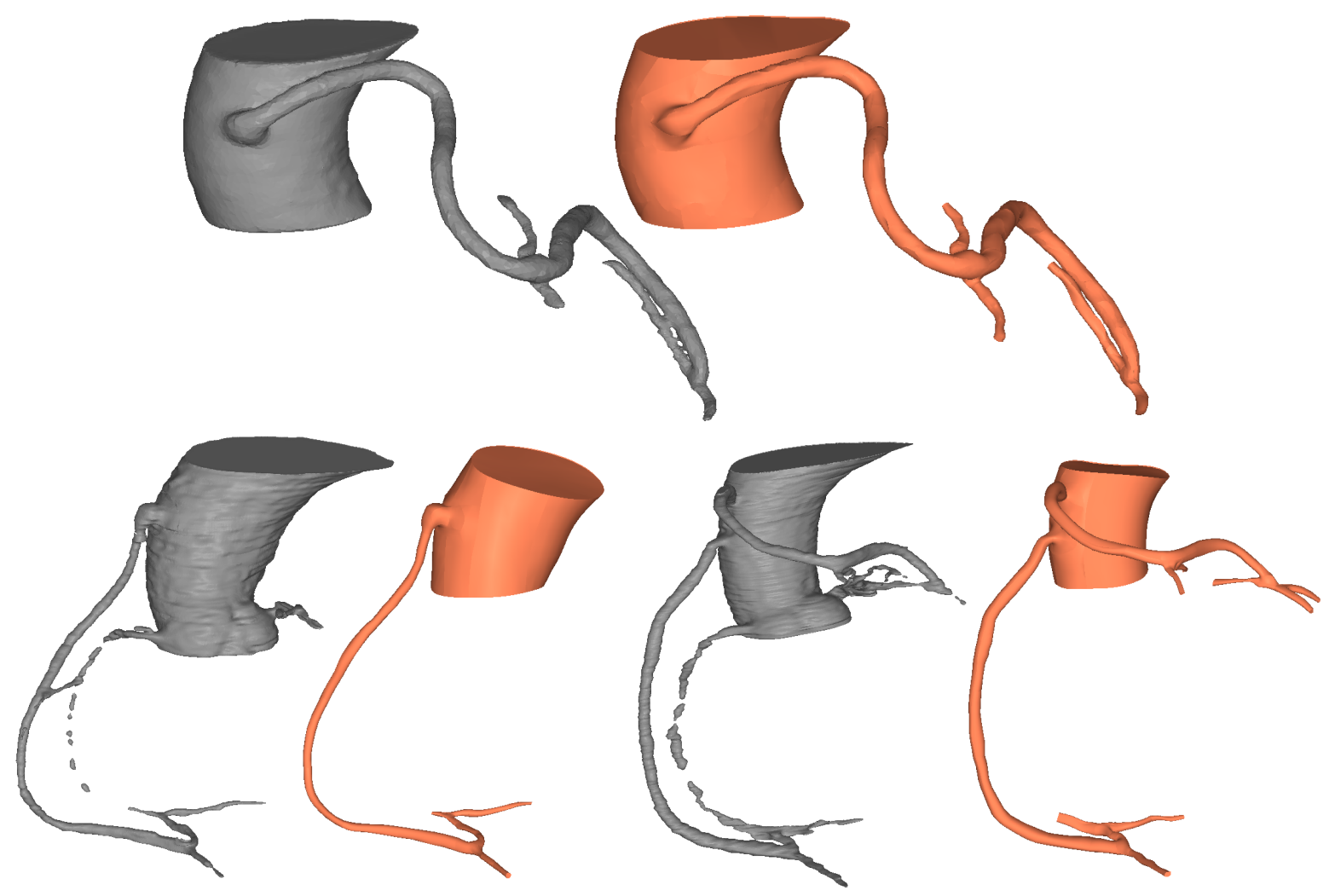

Figure 2. Primary reconstruction and smoothed model - aorto-coronary bypasses with sequential graft (top), individual graft (bottom left), and individual and sequential grafts (bottom right).

any possibility of turbulent flow in the ascending aorta, which is mainly associated with valve incompetence or artificial heart vales.

For the modelling of blood's complex rheological properties, we further introduce the shear-dependent Carreau-Yasuda model, which we have successfully applied in our previous study, [13],

$$
\eta(\dot{\gamma})=\eta_{\infty}+\left(\eta_{0}-\eta_{\infty}\right)\left[1+(\lambda \dot{\gamma})^{a}\right]^{\frac{n-1}{a}},
$$

where $\eta_{0}$ and $\eta_{\infty}$ are the zero and infinite shear viscosities, respectively, $\lambda$ is the characteristic relaxation time, $n$ is the flow index and $a$ is an approximation index. The five parameters appearing in the Carreau-Yasuda model (1) are usually determined by numerical fitting of experimental data. In this study, we adopt values mentioned in [3], i.e., $\eta_{\infty}=3.45 \cdot 10^{-3} \mathrm{~Pa} \cdot \mathrm{s}$, $\eta_{0}=56 \cdot 10^{-3} \mathrm{~Pa} \cdot \mathrm{s}, \lambda=1.902 \mathrm{~s}, a=1.25, n=0.22$. For the calculation of shear rate $\dot{\gamma}$ in Eq. (1), we apply following formula

$$
\dot{\gamma}=2 \sqrt{D_{I I}}
$$

where $D_{I I}$ denotes the second invariant of the rate of deformation tensor $\mathbf{D}=\frac{1}{2}\left(\nabla \boldsymbol{v}+(\nabla \boldsymbol{v})^{T}\right)$. Considering the fact that blood behaves as an incompressible fluid, the second invariant is defined as $D_{I I}=\frac{1}{2} d_{i j} d_{i j}, i, j=1,2,3$, where $d_{i j}$ are the components of the rate of deformation tensor D. For the sake of comparison, the molecular viscosity of the Newtonian fluid is set equal to the infinite shear viscosity $\eta_{\infty}$. 


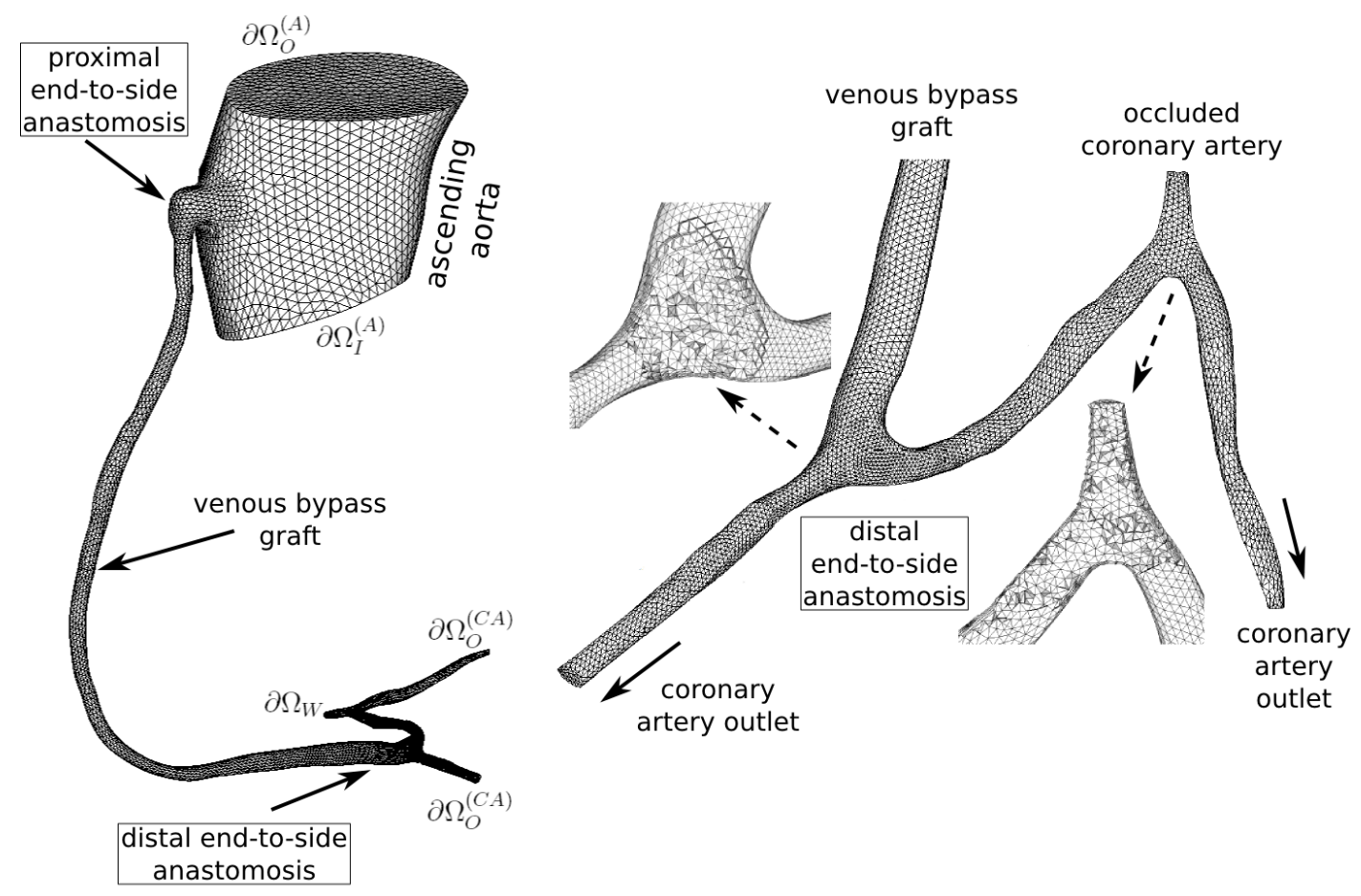

Figure 3. Unstructured tetrahedral computational mesh for the single aorto-coronary bypass model.

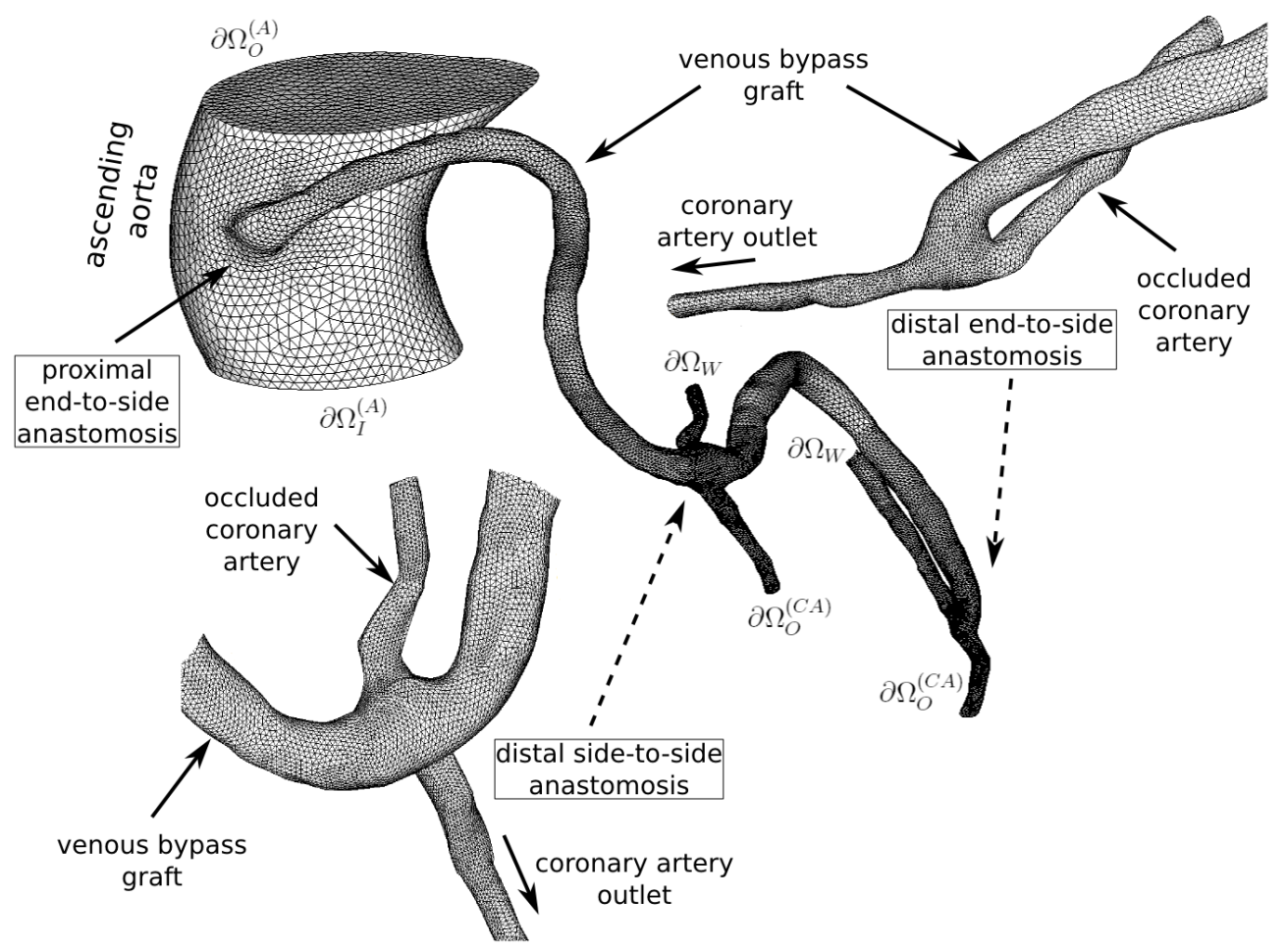

Figure 4. Unstructured tetrahedral computational mesh for the double aorto-coronary bypass model. 


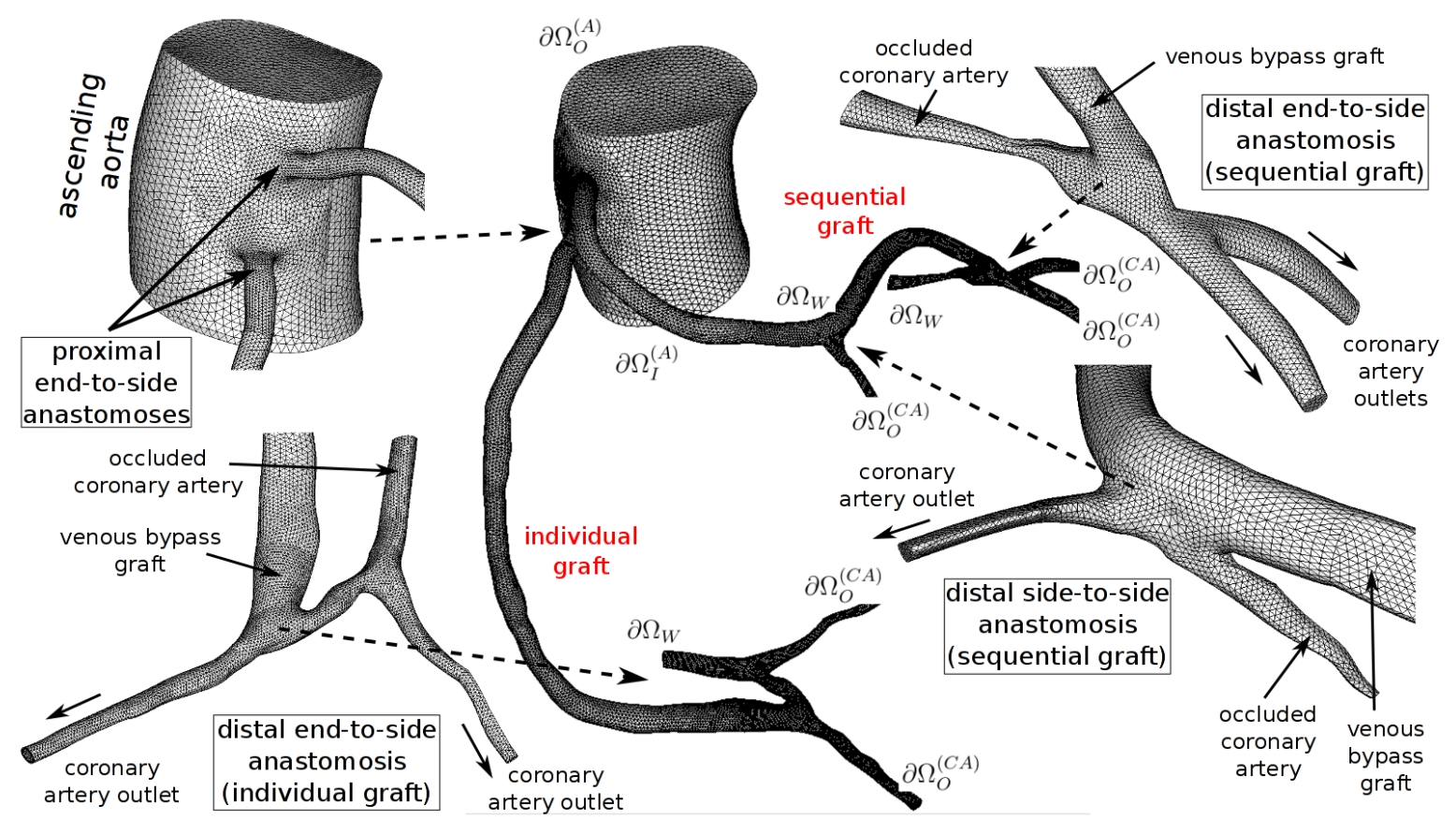

Figure 5. Unstructured tetrahedral computational mesh for the triple aorto-coronary bypass model.

In order to achieve pulsatile flow conditions approximating those of real blood flow in the ascending aorta, we consider time-dependent inlet flow rate $Q(t)$ and time-dependent outlet pressure $p(t)$. Both waveforms taken from [12] are shown in Fig. 6, where the aortic pressure is plotted in the medical units of millimeters of mercury $(1 \mathrm{mmHg}=133.333 \mathrm{~Pa})$. For reasons of comparison, same inlet and outlet values are going to be applied in the three aorto-coronary bypasses mentioned above.
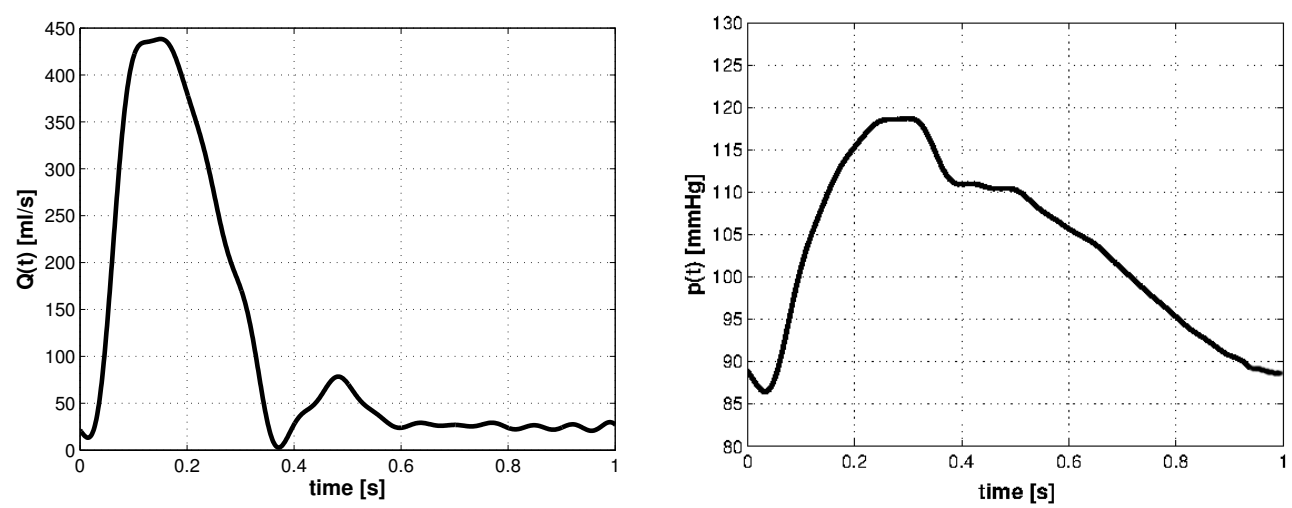

Figure 6. Time-dependent boundary conditions for the ascending aorta - inlet flow rate $Q(t)$ (left) and outlet pressure $p(t)$ (right), data taken from [12]

For the analysis of computed numerical results, we introduce two significant hemodynamical wall parameters - the cycle-averaged wall shear stress (WSS) and the oscillatory shear index (OSI) that are evaluated according to formulas mentioned in [15] and [7], respec- 
tively,

$$
\overline{\left|\boldsymbol{\tau}_{W}\right|}=\frac{1}{T} \int_{0}^{T}\left|\boldsymbol{\tau}_{W}\right| \mathrm{d} t, \quad \text { OSI }=\frac{1}{2}\left[1-\left|\int_{0}^{T} \boldsymbol{\tau}_{W} \mathrm{~d} t\right| \cdot\left(\int_{0}^{T}\left|\boldsymbol{\tau}_{W}\right| \mathrm{d} t\right)^{-1}\right],
$$

where $\left|\boldsymbol{\tau}_{W}\right|$ is the WSS magnitude and $T=1 \mathrm{~s}$ is the duration of one cardiac cycle, Fig. 6.

\section{MATHEMATICAL MODEL}

Let us consider a time interval $(0, \mathcal{T}), \mathcal{T}>0$ and a bounded three-dimensional computational domain $\Omega \subset \mathbf{R}^{3}$ with boundary $\partial \Omega=\partial \Omega_{I} \cup \partial \Omega_{O} \cup \partial \Omega_{W}$, where $\partial \Omega_{I}, \partial \Omega_{O}$ and $\partial \Omega_{W}$ denote the inlet, the outlet and the walls of the computational domain, respectively. In this study, the coronary blood flow is modelled as unsteady laminar isothermal flow of incompressible generalised Newtonian fluid that in the space-time cylinder $\Omega_{T}=\Omega \times(0, \mathcal{T})$ is mathematically described by the non-linear system of incompressible Navier-Stokes (NS) equations written in the non-dimensional form

$$
\begin{aligned}
\frac{\partial v_{i}}{\partial x_{i}} & =0 \\
\frac{\partial v_{i}}{\partial t}+\frac{\partial}{\partial x_{j}}\left(v_{i} v_{j}\right)+\frac{\partial p}{\partial x_{i}} & =\frac{1}{\operatorname{Re}} \frac{\partial}{\partial x_{j}}\left[\eta(\dot{\gamma})\left(\frac{\partial v_{i}}{\partial x_{j}}+\frac{\partial v_{j}}{\partial x_{i}}\right)\right] \quad \text { for } i, j=1,2,3,
\end{aligned}
$$

where $t \in(0, \mathcal{T})$ is the time, $v_{i}$ is the $i$-th component of the velocity vector $\boldsymbol{v}=\left[v_{1}, v_{2}, v_{3}\right]^{T}$ corresponding to the Cartesian component $x_{i}$ of the space variables vector $\boldsymbol{x}=\left[x_{1}, x_{2}, x_{3}\right]^{T} \in$ $\Omega, p$ is the pressure, $\operatorname{Re}$ is the reference Reynolds number and $\eta(\dot{\gamma})$ is the shear-dependent viscosity given by Eq. (1).

All variables appearing in Eqs. (4) - (5) are non-dimensionalized by the reference velocity $U_{r e f}>0$ and characteristic length $D_{\text {ref }}>0$. For the three bypass models considered in this study, the characteristic length value is chosen to be equal to the average aortic diameter $D_{\text {ref }} \equiv D_{(A)}=0.036 \mathrm{~m}$ and the reference velocity is stated as $U_{r e f}=4 Q_{0} /\left(\pi D_{r e f}^{2}\right)=$ $0.1592 \mathrm{~m} \cdot \mathrm{s}^{-1}$, where average aortic inlet flow rate is $Q_{0}=112.56 \cdot 10^{-6} \mathrm{~m}^{3} \cdot \mathrm{s}^{-1}$, see Fig. 6 (left). As for the reference Reynolds number, it is determined as $\operatorname{Re}=U_{\text {ref }} D_{\text {ref }} \varrho / \eta_{\text {ref }}=$ 1744.3 , where $\varrho=1050 \mathrm{~kg} \cdot \mathrm{m}^{-3}$ and $\eta_{\text {ref }} \equiv \eta_{\infty}=3.45 \cdot 10^{-3} \mathrm{~Pa} \cdot \mathrm{s}$. For the sake of completeness, reference pressure and reference time are computed as $p_{r e f}=\varrho U_{r e f}^{2}$ and $t_{r e f}=$ $D_{\text {ref }} / U_{\text {ref }}$, respectively.

\section{NUMERICAL METHOD}

The numerical solution of the non-linear time-dependent system of incompressible NS equations (4) - (5) is based on the projection method. In this study, the computation of velocity components $v_{i}^{n+1}$, which satisfy the divergence-free condition (4), employs the threestage fractional step scheme, [4]. In the first stage, intermediate velocity components $v_{i}^{*}$ are explicitly computed from the convective part of the NS equation (5) as

$$
\frac{v_{i}^{*}-v_{i}^{n}}{\Delta t}+\frac{\partial}{\partial x_{j}}\left(v_{i}^{n} v_{j}^{n}\right)=0, \quad i, j=1,2,3 .
$$


For the second stage of the fractional step scheme, the intermediate velocity components $\hat{v}_{i}$ are computed applying the unconditionally stable implicit Crank-Nicolson scheme to the viscous term of Eq. (5)

$$
\frac{\hat{v}_{i}-v_{i}^{*}}{\Delta t}=\frac{1}{2 \operatorname{Re}} \frac{\partial}{\partial x_{j}}\left[\eta(\dot{\gamma})\left(\frac{\partial\left(\hat{v}_{i}+v_{i}^{*}\right)}{\partial x_{j}}+\frac{\partial\left(\hat{v}_{j}+v_{j}^{*}\right)}{\partial x_{i}}\right)\right], \quad i, j=1,2,3 .
$$

Let us linearise the shear-dependent dynamic viscosity $\eta(\dot{\gamma})$ as $\bar{\eta}(\dot{\gamma})=\eta\left(\dot{\gamma}\left(v^{*}\right)\right)$ and introduce an auxiliary variable $d_{i j}=\frac{1}{2}\left(\frac{\partial v_{i}}{\partial x_{j}}+\frac{\partial v_{j}}{\partial x_{i}}\right)$, which in this case is equal to the components of the rate of deformation tensor $\mathbf{D}$ mentioned in Section 2 Then Eq. (7) can be rewritten as a system of two linear equations

$$
\begin{aligned}
\frac{\hat{v}_{i}-v_{i}^{*}}{\Delta t} & =\frac{1}{\operatorname{Re}} \frac{\partial}{\partial x_{j}}\left[\bar{\eta}(\dot{\gamma})\left(\hat{d}_{i j}+d_{i j}^{*}\right)\right], \\
\hat{d}_{i j} & =\frac{1}{2}\left(\frac{\partial \hat{v}_{i}}{\partial x_{j}}+\frac{\partial \hat{v}_{j}}{\partial x_{i}}\right) .
\end{aligned}
$$

In the third stage, pressure is used for the projection of the intermediate velocity vector $\hat{\boldsymbol{v}}$ onto a space of divergence-free velocity field to get the values of velocity and pressure at the next time level $(n+1)$. Hence, the velocity components $v_{i}^{n+1}$ are computed from

$$
\frac{v_{i}^{n+1}-\hat{v}_{i}}{\Delta t}+\frac{\partial p^{n+1}}{\partial x_{i}}=0, \quad i=1,2,3,
$$

where $p^{n+1}$ is computed from the Poisson equation for pressure

$$
\frac{\partial^{2} p^{n+1}}{\partial x_{i} \partial x_{i}}=\frac{1}{\Delta t} \frac{\partial \hat{v}_{i}}{\partial x_{i}}
$$

It can be easily shown that sum of Eqs. (6), (7) and (10) yields the approximation of NS equations of first order time accuracy. Finally, the whole algorithm of the fractional step method may be written for $i, j=1,2,3$ as follows

$$
\begin{aligned}
v_{i}^{*} & =v_{i}^{n}-\Delta t \frac{\partial}{\partial x_{j}}\left(v_{i}^{n} v_{j}^{n}\right) \\
\frac{\hat{v}_{i}}{\Delta t}-\frac{1}{\operatorname{Re}} \frac{\partial}{\partial x_{j}}\left(\bar{\eta}(\dot{\gamma}) \hat{d}_{i j}\right) & =\frac{v_{i}^{*}}{\Delta t}+\frac{1}{\operatorname{Re}} \frac{\partial}{\partial x_{j}}\left(\bar{\eta}(\dot{\gamma}) d_{i j}^{*}\right) \\
\hat{d}_{i j}-\frac{1}{2}\left(\frac{\partial \hat{v}_{i}}{\partial x_{j}}+\frac{\partial \hat{v}_{j}}{\partial x_{i}}\right) & =0 \\
\frac{\partial^{2} p^{n+1}}{\partial x_{i} \partial x_{i}} & =\frac{1}{\Delta t} \frac{\partial \hat{v}_{i}}{\partial x_{i}} \\
v_{i}^{n+1} & =\hat{v}_{i}-\Delta t \frac{\partial p^{n+1}}{\partial x_{i}}
\end{aligned}
$$

The space discretization of the system of Eqs. (12) - (16) is performed using the cellcentred finite volume method for hybrid unstructured tetrahedral grids. The idea of applying the hybrid unstructured grid for the numerical solution of time-dependent incompressible NS equations in 2D was introduced in [9]. The principle of this grid system lies in the coupling 


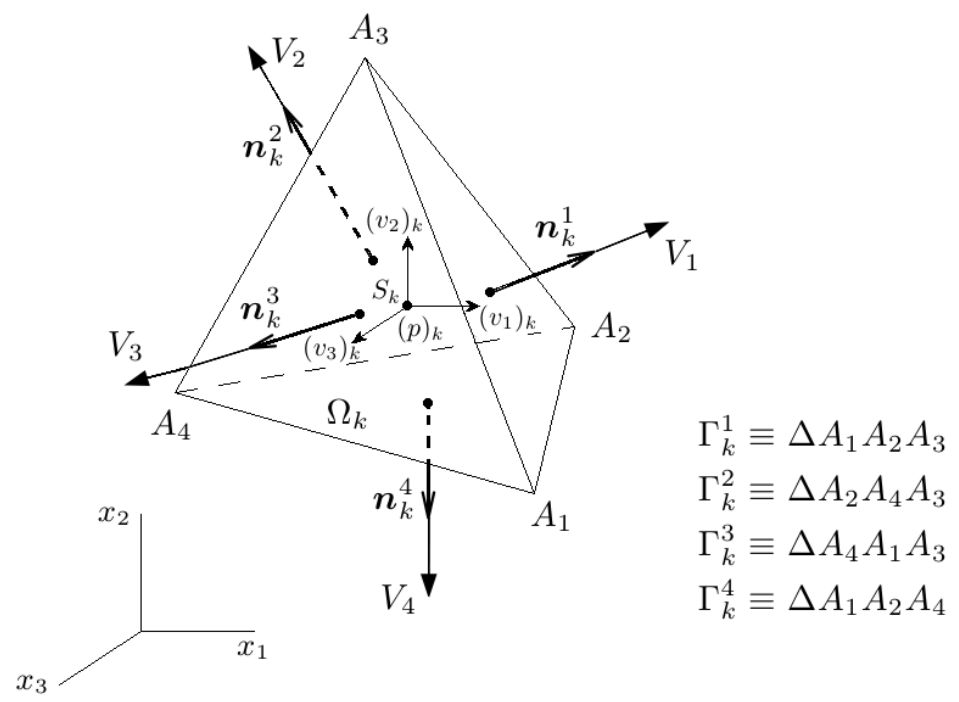

Figure 7. A tetrahedral control volume $\Omega_{k}=A_{1} A_{2} A_{3} A_{4}$ with boundary $\partial \Omega_{k}=\bigcup_{m=1}^{4} \Gamma_{k}^{m}$ belonging to the hybrid unstructured computational mesh.

between an interpolation method, which will be described later, and the non-staggered grid system. Being inspired with this idea, we consider in this study a control volume $\Omega_{k}$ in the form of tetrahedron, Fig. 7. The hybrid grid system defines the values of pressure and Cartesian velocity components in the centre of the control volume $\Omega_{k}$ and the values of facenormal velocity $V_{m}$, which has the direction of outward unit vector $\boldsymbol{n}_{k}^{m}$ normal to the $m$-th face $\Gamma_{k}^{m}$ of the control volume $\Omega_{k}$, is defined in the middle of the face $\Gamma_{k}^{m}$.

After the integration of Eqs. (12) - (16) over each control volume $\Omega_{k}$, Fig. 7, $k=$ $1,2, \ldots, N_{C V}$, where $N_{C V}$ is the number of control volumes within the hybrid unstructured tetrahedral computational mesh, after the introduction of integral average for an arbitrary flow quantity $\Phi$ over the control volume $\Omega_{k}$

$$
(\Phi)_{k}=\frac{1}{\left|\Omega_{k}\right|} \int_{\Omega_{k}} \Phi \mathrm{d} \Omega,
$$

where $\left|\Omega_{k}\right|$ is the volume of the tetrahedral control volume $\Omega_{k}$, and finally, after the application of the Gauss-Ostrogradsky theorem, which converts the volume integrals to surface integrals, we get

$$
\begin{gathered}
\left(v_{i}^{*}\right)_{k}=\left(v_{i}^{n}\right)_{k}-\frac{\Delta t}{\left|\Omega_{k}\right|} \oint_{\partial \Omega_{k}}\left(v_{j}^{n} \cdot{ }^{j} n_{k}\right) \cdot v_{i}^{n} \mathrm{~d} \Gamma \\
\frac{1}{\Delta t}\left(\hat{v}_{i}\right)_{k}-\frac{1}{\operatorname{Re}\left|\Omega_{k}\right|} \oint_{\partial \Omega_{k}} \bar{\eta}(\dot{\gamma}) \hat{d}_{i j} \cdot{ }^{j} n_{k} \mathrm{~d} \Gamma=\frac{1}{\Delta t}\left(v_{i}^{*}\right)_{k}+\frac{1}{\operatorname{Re}\left|\Omega_{k}\right|} \oint_{\partial \Omega_{k}} \bar{\eta}(\dot{\gamma}) d_{i j}^{*} \cdot{ }^{j} n_{k} \mathrm{~d} \Gamma \\
\left(\hat{d}_{i j}\right)_{k}-\frac{1}{2}\left(\oint_{\partial \Omega_{k}} \hat{v}_{i} \cdot{ }^{j} n_{k} \mathrm{~d} \Gamma+\oint_{\partial \Omega_{k}} \hat{v}_{j} \cdot{ }^{i} n_{k} \mathrm{~d} \Gamma\right)=0
\end{gathered}
$$




$$
\begin{gathered}
\oint_{\partial \Omega_{k}} \frac{\partial p^{n+1}}{\partial \boldsymbol{n}_{k}} \mathrm{~d} \Gamma=\frac{1}{\Delta t} \oint_{\partial \Omega_{k}} \hat{v}_{i} \cdot{ }^{i} n_{k} \mathrm{~d} \Gamma \\
\left(v_{i}^{n+1}\right)_{k}=\left(\hat{v}_{i}\right)_{k}-\frac{\Delta t}{\left|\Omega_{k}\right|} \oint_{\partial \Omega_{k}} p^{n+1} \cdot{ }^{i} n_{k} \mathrm{~d} \Gamma,
\end{gathered}
$$

where ${ }^{i} n_{k}$ is the $i$-th component of the outward unit vector $\boldsymbol{n}_{k}=\left[{ }^{1} n_{k},{ }^{2} n_{k},{ }^{3} n_{k}\right]^{T}$ normal to the boundary $\partial \Omega_{k}$ of the tetrahedral control volume $\Omega_{k}$, Fig. 7. In order to achieve the satisfaction of the continuity equation for the normal velocity $V=v_{i} \cdot{ }^{i} n_{k}$, the system of Eqs. (18) - (22) is completed with following equation

$$
V^{n+1}=\hat{V}-\Delta t \frac{\partial p^{n+1}}{\partial \boldsymbol{n}_{k}}
$$

This equation defines the normal velocity $V^{n+1}$ at the time level $(n+1)$ having the direction of the outward unit vector $\boldsymbol{n}_{k}$ normal to the boundary $\partial \Omega_{k}$ of the control volume $\Omega_{k}$. For the intermediate normal velocity $\hat{V}$, it is valid that $\hat{V}=\hat{v}_{i} \cdot{ }^{i} n_{k}$.

Further, we perform the approximation of surface integrals in the system of Eqs. (18) (22). Firstly, each integral is replaced by the sum of integrals over each face $\Gamma_{k}^{m}$ of the control volume $\Omega_{k}$, Fig. 7, and then approximated by the midpoint rule

$$
\oint_{\partial \Omega_{k}} \Phi \mathrm{d} \Gamma=\sum_{m=1}^{4} \int_{\Gamma_{k}^{m}} \Phi \mathrm{d} \Gamma \approx \sum_{m=1}^{4} \Phi_{m}\left|\Gamma_{k}^{m}\right|,
$$

where $\left|\Gamma_{k}^{m}\right|, m=1, \ldots, 4$ is the area of the $m$-th face $\Gamma_{k}^{m}$ of the control volume $\Omega_{k}$ and $\Phi_{m}$ is the value of an arbitrary flow quantity at the integration point at the same face. The interpolation process needed for the determination of $\Phi_{m}$ at the $m$-th face $\Gamma_{k}^{m}$ of the control volume $\Omega_{k}$ will be described later. Using Eq. (24), the system of Eqs. (18) - (23) is modified as follows

$$
\begin{gathered}
\left(v_{i}^{*}\right)_{k}=\left(v_{i}^{n}\right)_{k}-\frac{\Delta t}{\left|\Omega_{k}\right|} \sum_{m=1}^{4}\left(\left.V_{m}^{n} \cdot v_{i m}^{n}\right|_{\text {upwind }}\right)\left|\Gamma_{k}^{m}\right| \\
\frac{\left(\hat{v}_{i}\right)_{k}}{\Delta t}-\frac{1}{\operatorname{Re}\left|\Omega_{k}\right|} \sum_{m=1}^{4} \bar{\eta}(\dot{\gamma})^{m} \hat{d}_{i j}^{m} \cdot{ }^{j} n_{k}^{m}\left|\Gamma_{k}^{m}\right|=\frac{\left(v_{i}^{*}\right)_{k}}{\Delta t}+\frac{1}{\operatorname{Re}\left|\Omega_{k}\right|} \sum_{m=1}^{4} \bar{\eta}(\dot{\gamma})^{m} d_{i j}^{* m} \cdot{ }^{j} n_{k}^{m}\left|\Gamma_{k}^{m}\right| \\
\left(\hat{d}_{i j}\right)_{k}-\frac{1}{2}\left(\sum_{m=1}^{4} \hat{v}_{i m} \cdot{ }^{j} n_{k}^{m}\left|\Gamma_{k}^{m}\right|+\sum_{m=1}^{4} \hat{v}_{j m} \cdot{ }^{i} n_{k}^{m}\left|\Gamma_{k}^{m}\right|\right)=0 \\
\sum_{m=1}^{4} \frac{\partial p^{n+1}}{\partial \boldsymbol{n}_{k}^{m}}\left|\Gamma_{k}^{m}\right|=\frac{1}{\Delta t} \sum_{m=1}^{4} \hat{v}_{i m} \cdot{ }^{i} n_{k}^{m}\left|\Gamma_{k}^{m}\right| \equiv \frac{1}{\Delta t} \sum_{m=1}^{4} \hat{V}_{m}\left|\Gamma_{k}^{m}\right| \\
\left(v_{i}^{n+1}\right)_{k}=\left(\hat{v}_{i}\right)_{k}-\frac{\Delta t}{\left|\Omega_{k}\right|} \sum_{m=1}^{4} p_{m}^{n+1} \cdot{ }^{i} n_{k}^{m}\left|\Gamma_{k}^{m}\right| \\
V_{m}^{n+1}=\hat{V}_{m}-\Delta t \frac{\partial p^{n+1}}{\partial \boldsymbol{n}_{k}^{m}}
\end{gathered}
$$


where ${ }^{i} n_{k}^{m}$ is the $i$-th component of the outward unit vector $\boldsymbol{n}_{k}^{m}=\left[{ }^{1} n_{k}^{m},{ }^{2} n_{k}^{m},{ }^{3} n_{k}^{m}\right]^{T}$ normal to the $m$-th face $\Gamma_{k}^{m}$ of the control volume $\Omega_{k}$ and for the intermediate face-normal velocity $\hat{V}_{m}$ at the $m$-th face $\Gamma_{k}^{m}$ of the control volume $\Omega_{k}$, it is valid that $\hat{V}_{m}=\hat{v}_{i m} \cdot{ }^{i} n_{k}^{m}$. Note that the values of face-normal velocity $V_{m}^{n+1}$ computed with the help of Eq. (30) are used as values of face-normal velocity $V_{m}^{n}$ in Eq. (25) at the next time level.

Explicit schemes are known for their disadvantage in the form of restricted time steps. The CFL stability condition imposed on the time step size becomes essential when it is applied for grids with large differences in cell size, e.g., in complex geometries. In this case, the efficiency of explicit schemes is lost, since the cell with the most restrictive local time step determines the size of the global time step for all grid cells. One of possible solutions to this problem lies in the application of the well-known local time-stepping method. This method, whose approach is also employed in our developed solver, enables each cell of the computational grid to run with its own time step in a time-consistent manner.

\section{Interpolation method}

To perform numerical computations according to Eqs. (25) - (30), it is necessary to determine values of $\left.v_{i m}^{n}\right|_{\text {upwind }}, \hat{v}_{i m}, p_{m}^{n+1}$ and derivatives $\frac{\partial \hat{v}_{i}}{\partial \boldsymbol{n}_{k}^{m}}, \frac{\partial v_{i}^{*}}{\partial \boldsymbol{n}_{k}^{m}}, \frac{\partial p^{n+1}}{\partial \boldsymbol{n}_{k}^{m}}$ at the $m$-th face $\Gamma_{k}^{m}$ of the control volume $\Omega_{k}$. The value of $\left.v_{i m}^{n}\right|_{\text {upwind }}$ is computed by the upwind scheme, whose first order accuracy is increased by linear reconstruction with Barth's limiter,

$$
\left.v_{i m}^{n}\right|_{\text {upwind }}= \begin{cases}\left(v_{i}^{n}\right)_{L}+\sigma_{L}^{B a r t h} \cdot \frac{\partial\left(v_{i}^{n}\right)_{L}}{\partial x_{j}} \cdot r_{j L}, & V_{m}^{n}>0, \\ \left(v_{i}^{n}\right)_{R}+\sigma_{R}^{B a r t h} \cdot \frac{\partial\left(v_{i}^{n}\right)_{R}}{\partial x_{j}} \cdot r_{j R}, & V_{m}^{n} \leq 0,\end{cases}
$$

where $\sigma^{\text {Barth }} \in[0,1]$ is the Barth's limiter, [2], and vectors $\boldsymbol{r}_{L}, \boldsymbol{r}_{R}$ are denoted in Fig. 8 (left). Further, the value $\Phi_{m}$ of an arbitrary flow quantity $\Phi$ at the mid-point $\mathrm{O}$ of the $m$-th face $\Gamma_{k}^{m}$, Fig. 8 (right), can be stated with the help of second order accurate linear interpolation from values $(\Phi)_{k}$ and $(\Phi)_{l_{m}}$ defined in cell-centres $S_{k}$ and $S_{l_{m}}$ of two adjacent control volumes $\Omega_{k}$ and $\Omega_{l_{m}}$, respectively,

$$
\Phi_{m}=(\Phi)_{k}+\frac{(\Phi)_{l_{m}}-(\Phi)_{k}}{\gamma_{k}+\gamma_{l_{m}}} \cdot \gamma_{k}=\frac{\gamma_{l_{m}}(\Phi)_{k}+\gamma_{k}(\Phi)_{l_{m}}}{\gamma_{k}+\gamma_{l_{m}}}
$$

where $\gamma_{k}$ and $\gamma_{l_{m}}$ are the minimal distances to the cell-face $\Gamma_{k}^{m}$ from cell-centres $S_{k}$ and $S_{l_{m}}$ of the adjacent control volumes $\Omega_{k}$ and $\Omega_{l_{m}}$, respectively, Fig. 8 (right). The derivative of flow quantity $\Phi$ in the direction of the outward unit vector $\boldsymbol{n}_{k}^{m}$ normal to the $m$-th face $\Gamma_{k}^{m}$ of the control volume $\Omega_{k}$, is approximated at the mid-point $\mathrm{O}$ of the face $\Gamma_{k}^{m}$, Fig. 8 (right), as

$$
\left.\frac{\partial \Phi}{\partial \boldsymbol{n}_{k}^{m}}\right|_{\Gamma_{k}^{m}} \approx \frac{(\Phi)_{l_{m}}-(\Phi)_{k}}{\gamma_{k}+\gamma_{l_{m}}} .
$$

A crucial part of the interpolation method is the application of Eq. (33) to the normal derivative in Eqs. (28) and (30). In this way, it is ensured that the face-normal velocity $V_{m}^{n+1}$ satisfies the continuity equation at the time level $(n+1)$ exactly, see Eq. (39). However, in general velocities $\left(v_{i}^{n+1}\right)_{k}$ in cell-centres of control volumes $\Omega_{k}$ do not satisfy the continuity equation.

Regarding the implementation of non-dimensional boundary conditions at the boundary $\partial \Omega$ of the computational domain $\Omega \subset \mathbf{R}^{3}$, three boundary types are considered in this study: 

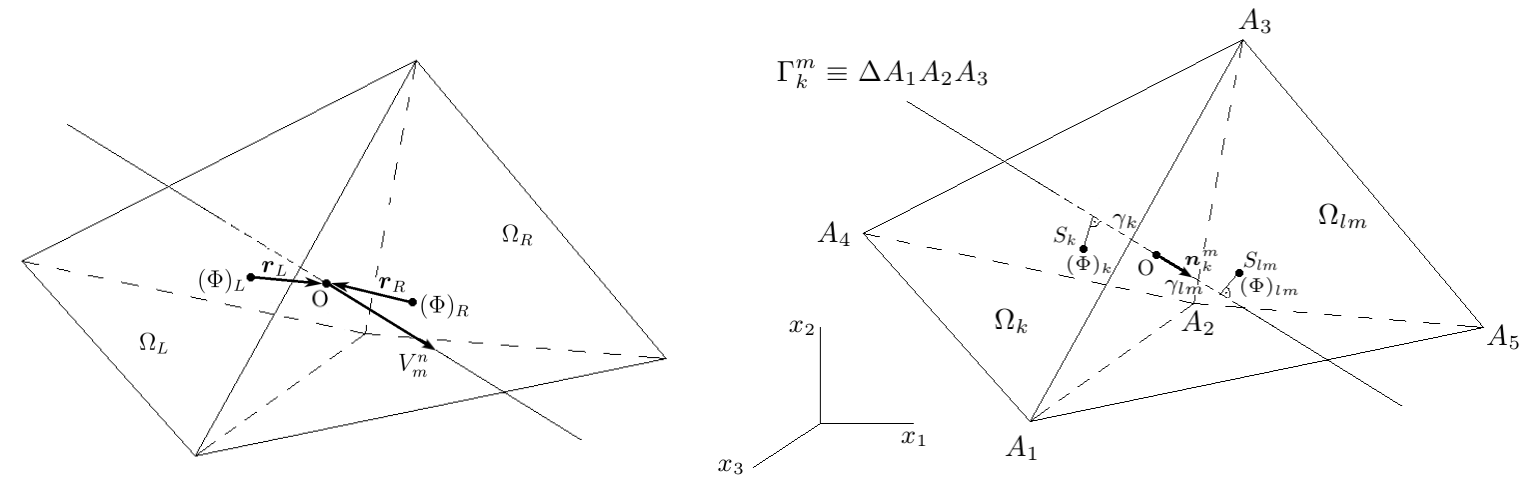

Figure 8. Definition of the vectors $\boldsymbol{r}_{L}$ and $\boldsymbol{r}_{R}$ for two adjacent tetrahedral control volumes $\Omega_{L}$ and $\Omega_{R}$ (left). Two adjacent tetrahedral control volumes $\Omega_{k}=A_{1} A_{2} A_{3} A_{4}$ and $\Omega_{l_{m}}=A_{1} A_{2} A_{3} A_{5}$ with their contact face $\Gamma_{k}^{m}=\Delta A_{1} A_{2} A_{3}$ (right).

- inlet $\Gamma_{k}^{m} \subset \partial \Omega_{I}$ - In this case, Dirichlet boundary conditions for the velocity components $v_{i m}$ and the auxiliary variable $d_{i j}^{m}$ are prescribed

$$
v_{i m}=v_{i I}, \quad d_{i j}^{m} \cdot{ }^{j} n=0 .
$$

The value of face-normal velocity $V_{m}^{I}$ at the face $\Gamma_{k}^{m}$ is computed as $V_{m}^{I}=v_{i I} \cdot{ }^{i} n_{k}^{m}$, where values $v_{i I}$ are given according to Section 5 . For the normal derivative of the pressure $p^{n+1}$ at the face $\Gamma_{k}^{m}$, we prescribe

$$
\left.\frac{\partial p^{n+1}}{\partial \boldsymbol{n}_{k}^{m}}\right|_{\Gamma_{k}^{m}}=0 .
$$

- rigid and impermeable wall $\Gamma_{k}^{m} \subset \partial \Omega_{W}$ - Velocity components $v_{i m}$ at the face $\Gamma_{k}^{m}$ are set equal to zero

$$
v_{i m}=0,
$$

leading to zero value of the face-normal velocity $V_{m}^{W}=v_{i m} \cdot{ }^{i} n_{k}^{m}=0$ at the face $\Gamma_{k}^{m}$. For the auxiliary variable $d_{i j}^{m}$, we apply the Dirichlet boundary condition in the following form

$$
d_{i j}^{m} \cdot{ }^{j} n=0 .
$$

Further, zero normal derivative of the pressure $p^{n+1}(35)$ is prescribed at the wall.

- outlet $\Gamma_{k}^{m} \subset \partial \Omega_{O}$ - Following type of boundary condition is stated

$$
p_{m} \boldsymbol{n}_{k}^{m}-\frac{1}{\operatorname{Re}} 2 \bar{\eta}(\dot{\gamma})^{m} d_{i j}^{m} \cdot{ }^{j} n_{k}^{m}=p_{O} \boldsymbol{n}_{k}^{m},
$$

where $p_{O}$ is the given value of the outlet pressure, see Section 5.

Substituting the derivative $\frac{\partial p^{n+1}}{\partial \boldsymbol{n}_{k}^{m}}$ in Eq. (28) with Eq. (30), we get

$$
\begin{aligned}
\sum_{m=1}^{4} \frac{\partial p^{n+1}}{\partial \boldsymbol{n}_{k}^{m}}\left|\Gamma_{k}^{m}\right| & =\frac{1}{\Delta t} \sum_{m=1}^{4}\left(\hat{V}_{m}-V_{m}^{n+1}\right)\left|\Gamma_{k}^{m}\right|=\frac{1}{\Delta t} \sum_{m=1}^{4} \hat{V}_{m}\left|\Gamma_{k}^{m}\right| \\
& \Longrightarrow \sum_{m=1}^{4} V_{m}^{n+1}\left|\Gamma_{k}^{m}\right|=0,
\end{aligned}
$$


i.e., face-normal velocities $V_{m}^{n+1}$ satisfy the continuity equation exactly. At this point, let us mention that at the outlet boundary $\partial \Omega_{O}$, i.e., at the face $\Gamma_{k}^{m}$ of the control volume $\Omega_{k}$, where $\Gamma_{k}^{m} \subset \partial \Omega_{O}$, values $\frac{\partial p^{n+1}}{\partial \boldsymbol{n}_{k}^{m}}$ are unknown. In order to ensure the satisfaction of the continuity equation (39) for this control volume $\Omega_{k}$, it is necessary to compute the face-normal velocity $V_{m}^{n+1}$ at the face $\Gamma_{k}^{m} \subset \partial \Omega_{O}$ as

$$
V_{m_{O}}^{n+1}=-\frac{1}{\left|\Gamma_{k}^{m_{O}}\right|} \sum_{\substack{m=1 \\ m \neq m_{O}}}^{4} V_{m}^{n+1}\left|\Gamma_{k}^{m}\right|
$$

where $m_{O}$ is the index of the outlet face $\Gamma_{k}^{m_{O}}$ of the control volume $\Omega_{k}$. For the whole computational domain $\Omega \subset \mathbf{R}^{3}$ at the time $t=0$, following initial conditions are used

$\left(v_{i}^{0}\right)_{k}=\frac{1}{\left|\Omega_{k}\right|} \int_{\Omega_{k}} v_{i}(\boldsymbol{x}, 0) \mathrm{d} \Omega=0, \quad\left(p^{0}\right)_{k}=\frac{1}{\left|\Omega_{k}\right|} \int_{\Omega_{k}} p(\boldsymbol{x}, 0) \mathrm{d} \Omega=p_{\text {initial }}, k=1,2, \ldots, N_{C V}$, where $p_{\text {initial }}$ is a non-dimensional value of static pressure.

In this study, the above described numerical method was implemented in the MATLAB software. The developed computational code was verified for the steady flow of an incompressible fluid in a straight tube by prescribing a constant velocity profile at the inlet and by comparing the obtained numerical results with the well-known analytical solution for the Poiseuille flow.

\section{BOUNDARY CONDITIONS}

In accordance with the boundaries of the computational domain labelled in Figs. 3 5 for the three relevant bypass models, the numerical simulations of the pulsatile Newtonian and non-Newtonian blood flow were carried out with following boundary values:

- aortic inlet $\partial \Omega_{I}^{(A)}$ - constant time-dependent velocity profile $\left|\boldsymbol{v}_{I}\right|$ computed from the flow rate waveform $Q(t)$, Fig. 6 (left). The assumption of the non-parabolic velocity profile is based on observations published in [10], where "blunted" velocity profiles were measured for the ascending aorta with native heart valves;

- aortic outlet $\partial \Omega_{O}^{(A)}$ - outlet pressure $p_{O}$ corresponding to the time-dependent pressure $p(t)$ shown in Fig. 6 (right);

- coronary outlets $\partial \Omega_{O}^{(C A)}$ - constant outlet pressure $p_{O}$ equal to the average arterial pressure of $12000 \mathrm{~Pa}$;

- rigid and impermeable walls and occluded arteries $\partial \Omega_{W}$ - non-slip boundary condition.

Note that the boundary values mentioned above are, for the computation, non-dimensionalized using the reference values mentioned in Section 3. The implementation of the boundary conditions in the numerical code is described in detail in Section 4.

\section{NUMERICAL RESULTS AND DISCUSSION}

The transient numerical simulations were run on a computer with Intel Xeon X5460 $(3.16 \mathrm{GHz})$ processor and 64GiB RAM. The CPU times for the single, double and triple bypass model after reaching three full cardiac cycles were approximately 6,8 and 15 days, 
respectively. Distinct differences in CPU times between the Newtonian and non-Newtonian flow computations were not observed. For the terminology used in the remainder of this section in relation to the relevant parts of each bypass model, we refer the reader to Figs. 3-5.

\subsection{Single bypass}

Firstly, let us analyse the velocity profiles of the non-Newtonian blood flow in the single aorto-coronary bypass, Fig. 9, for two selected time instants, corresponding to the systole and diastole, respectively. During the systolic phase $\left(t_{1}=0.16 \mathrm{~s}\right)$, Fig. 9 (left), the graft's proximal anastomosis becomes exposed to the increased flow rate in the aorta. Although the skewed velocity profiles at the graft entrance may indicate incoming blood flow, the real graft filling occurs later, mainly during the diastolic phase $\left(t_{2}=0.47 \mathrm{~s}\right)$, Fig. 9 (right). In this case, the velocity increase observed along the individual graft is also accompanied by skewed or otherwise shaped velocity profiles that are a result of the out-of-plane geometry and of the graft's winding around the heart, Fig. 9b. At the distal end-to-side anastomosis, Fig. 9c, the
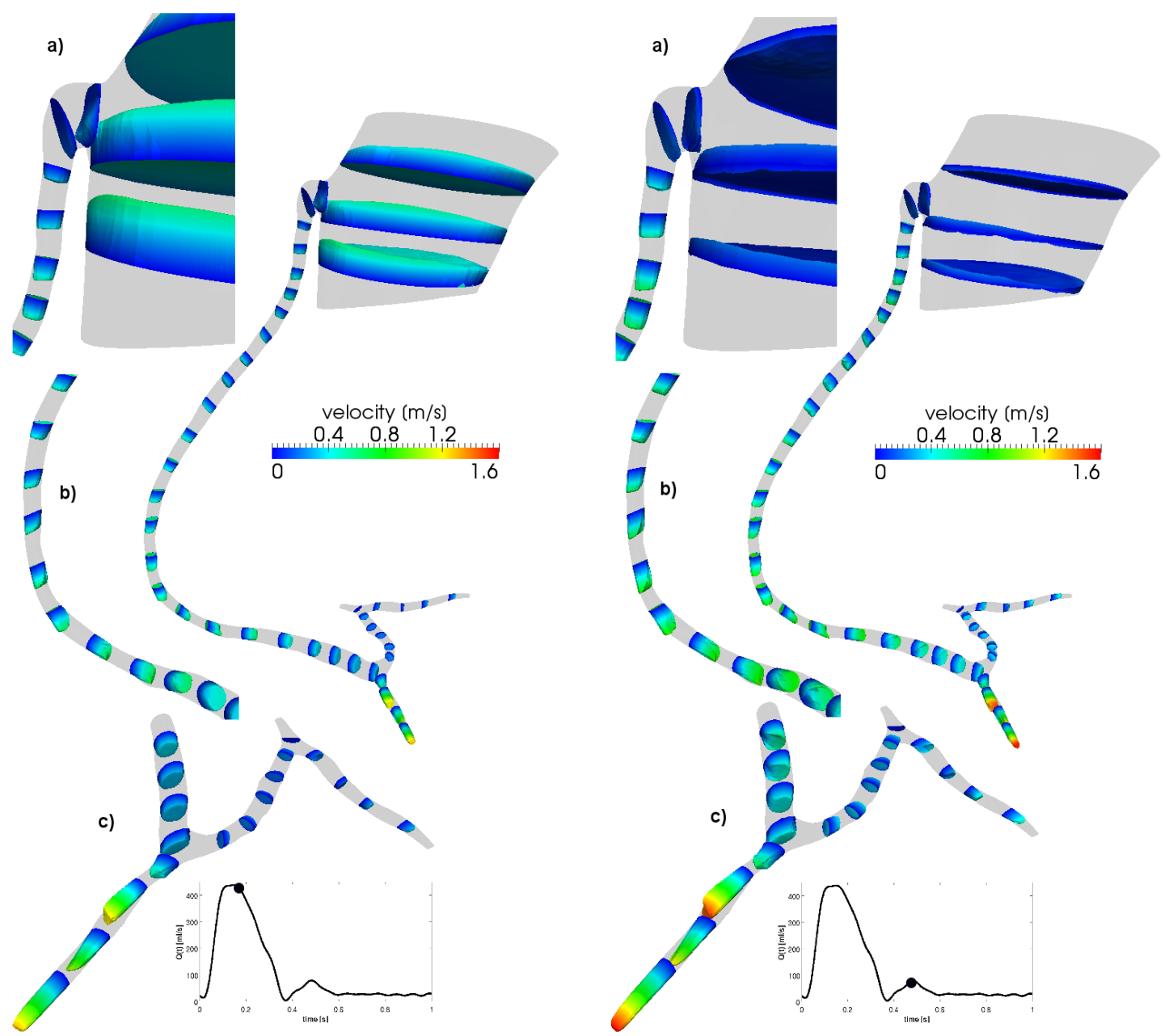

Figure 9. Non-Newtonian blood flow in the single aorto-coronary bypass model - velocity profiles at selected cross-sections at $t_{1}=0.16 \mathrm{~s}$ (left) and $t_{2}=0.47 \mathrm{~s}($ right $)$ with detailed views at the (a) proximal anastomosis, (b) individual graft, (c) coronary arteries with the distal anastomosis. 
incoming blood flow seems to prefer the closer branch of the coronary artery more than the second one.

The comparison between the Newtonian and non-Newtonian blood flow in the single bypass is illustrated by the distributions of the cycle-averaged WSS and OSI in Figs. 10 11, respectively. At this point, note the lowered value range in Fig. 10, which is chosen according to conclusions mentioned in [6] and [7]. Namely, that low WSS, as compared to the normal range between $1-2 \mathrm{~Pa}$ in healthy arteries, is one of the confirmed triggers of vessel remodelling, plaque growth and intimal thickening. In light of this fact, we will further assess the resulting shear distribution, which at both anastomoses seems to be very similar for Newtonian and non-Newtonian flows, Fig. 10. One of the distinct areas with extremely low shear is situated at the entrance of the graft, where it is caused by a large recirculation zone that is present there most of the cardiac cycle. This negative stimulation of the proximal suture line is also confirmed by the high OSI shown in Fig. 11. At the distal anastomosis, shear values below $1 \mathrm{~Pa}$ are observed at the heel and the arterial floor in accordance with the sites of intimal hyperplasia displayed in Fig. 1. In this case, the critical shear stress also shows a oscillatory tendency as is apparent from the OSI distribution in Fig. 11.

\subsection{Double bypass}

Velocity profiles in the double aorto-coronary bypass model are shown in Fig. 12 for the time instants $t_{1}=0.16 \mathrm{~s}$ (top) and $t_{2}=0.47 \mathrm{~s}$ (bottom). In comparison to the previous bypass with individual graft, the double bypass demonstrates higher blood flow and velocity
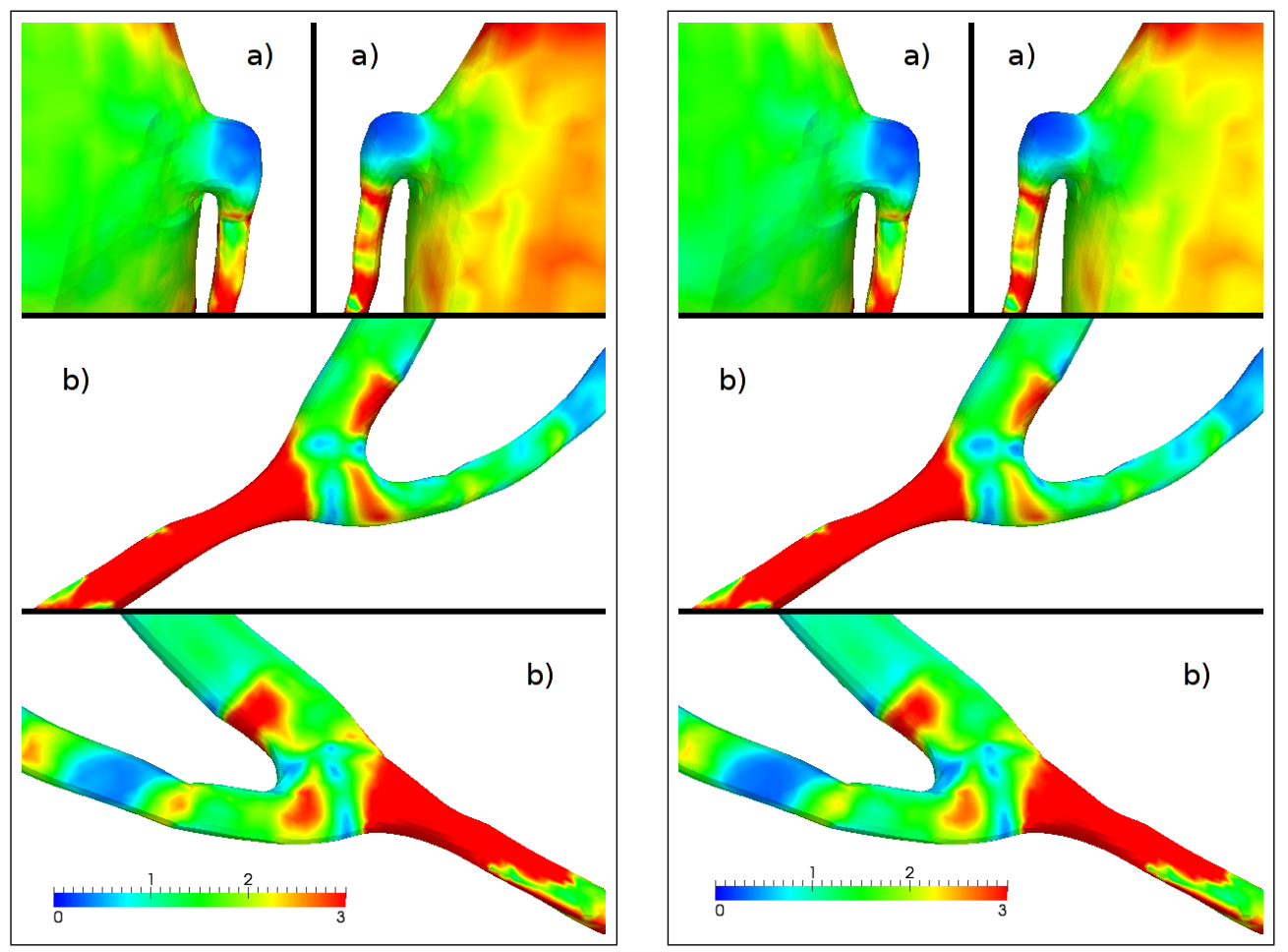

Figure 10. Single aorto-coronary bypass model - distribution of the cycle-averaged WSS magnitude for the Newtonian (left) and non-Newtonian flow (right) with detailed views at the (a) proximal and (b) distal anastomoses of the individual graft. 

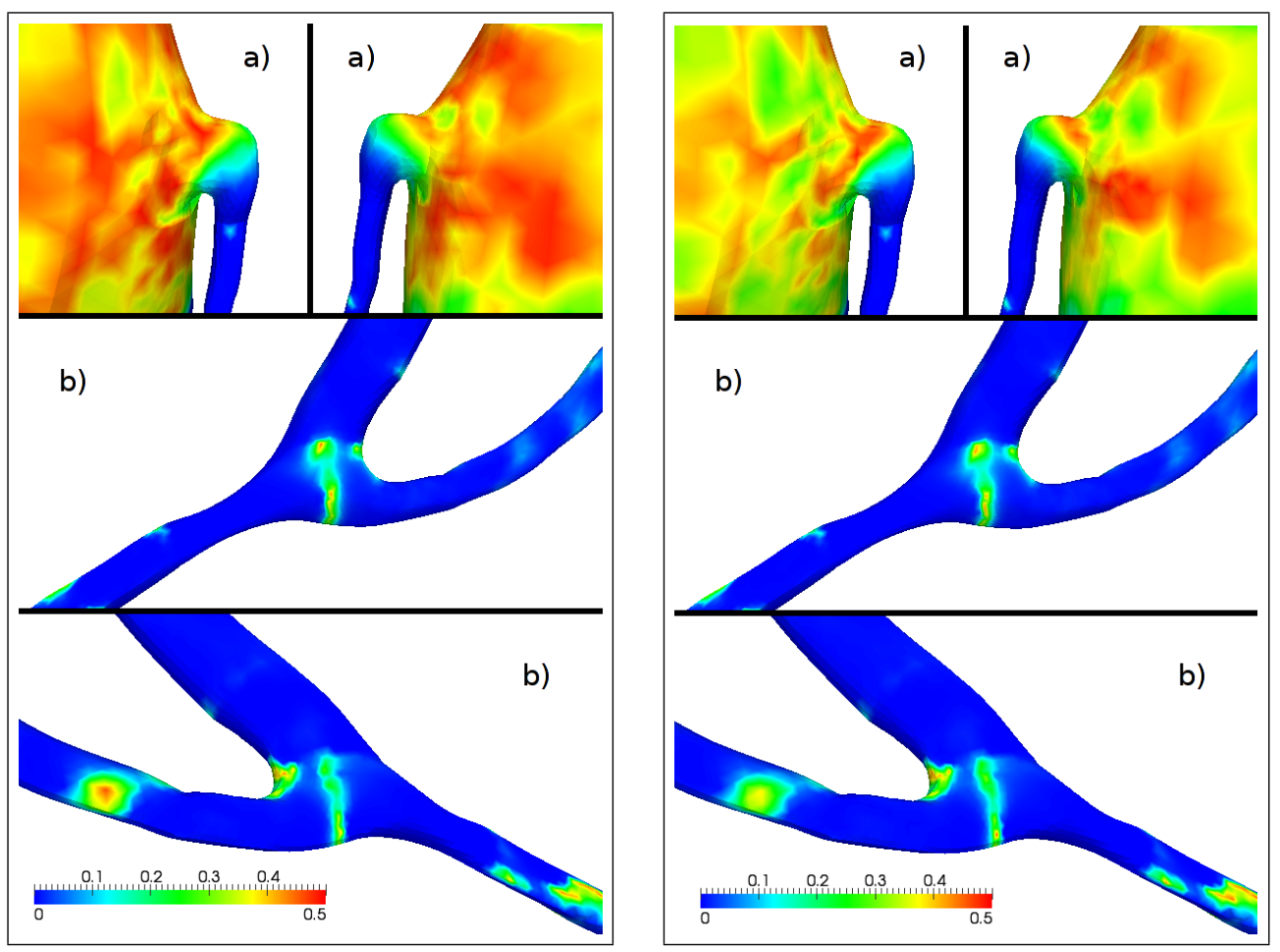

Figure 11. Single aorto-coronary bypass model - distribution of the OSI for the Newtonian (left) and non-Newtonian flow (right) with detailed views at the (a) proximal and (b) distal anastomoses of the individual graft.

in the proximal (pre-anastomotic) segment of the sequential graft. This observation is in accordance with intraoperative studies, e.g., [14], which imply higher patency rates for the bypasses with side-to-side anastomoses. The influence of the out-of-plane geometry on the shape of the velocity profiles is well apparent in the case of the side-to-side anastomosis, Fig. 12b. Here the incoming flow is strongly skewed towards the lower wall, facilitating the blood filling of the connected coronary artery during diastole. For the numerical simulation, we assumed that both coronary arteries were occluded with no inflow. As a result, the preanastomotic segments of the coronary arteries at the distal anastomoses are filled with large 'dead-water' zones, Fig. 12b-c.

The distribution of the cycle-averaged WSS magnitude at the distal anastomoses is displayed in Fig. 13 for the Newtonian and non-Newtonian blood flow. Once again, the WSS patterns of the two flows are almost identical, with only a few subtle differences. In accordance with the flow fields in Fig. 12, the presence of low shear values $(<1 \mathrm{~Pa})$ is primarily associated with the occluded coronary segments or with parts where the graft's out-of-plane geometry is particularly noticeable, see Fig. 13b. Considering the possibility of shear oscillations at the distal anastomoses, Fig. 14, the sequential bypass graft shows similar patterns as the individual one for the Newtonian and non-Newtonian blood flow. Probably the only exception can be found in the side-to-side anastomosis, Fig. 14b, where the OSI level demonstrates a considerable increase within the bypass graft. 


\subsection{Triple bypass}

The triple bypass as a combination of the individual and sequential graft shows features similar to the single and double bypasses, which were discussed in the previous two sections. For example, blunter velocity profiles are typical for the systolic phase of the cardiac cycle, Fig. 15b-c (left), as compared to the skewed ones during the diastole, Fig. 15b-c
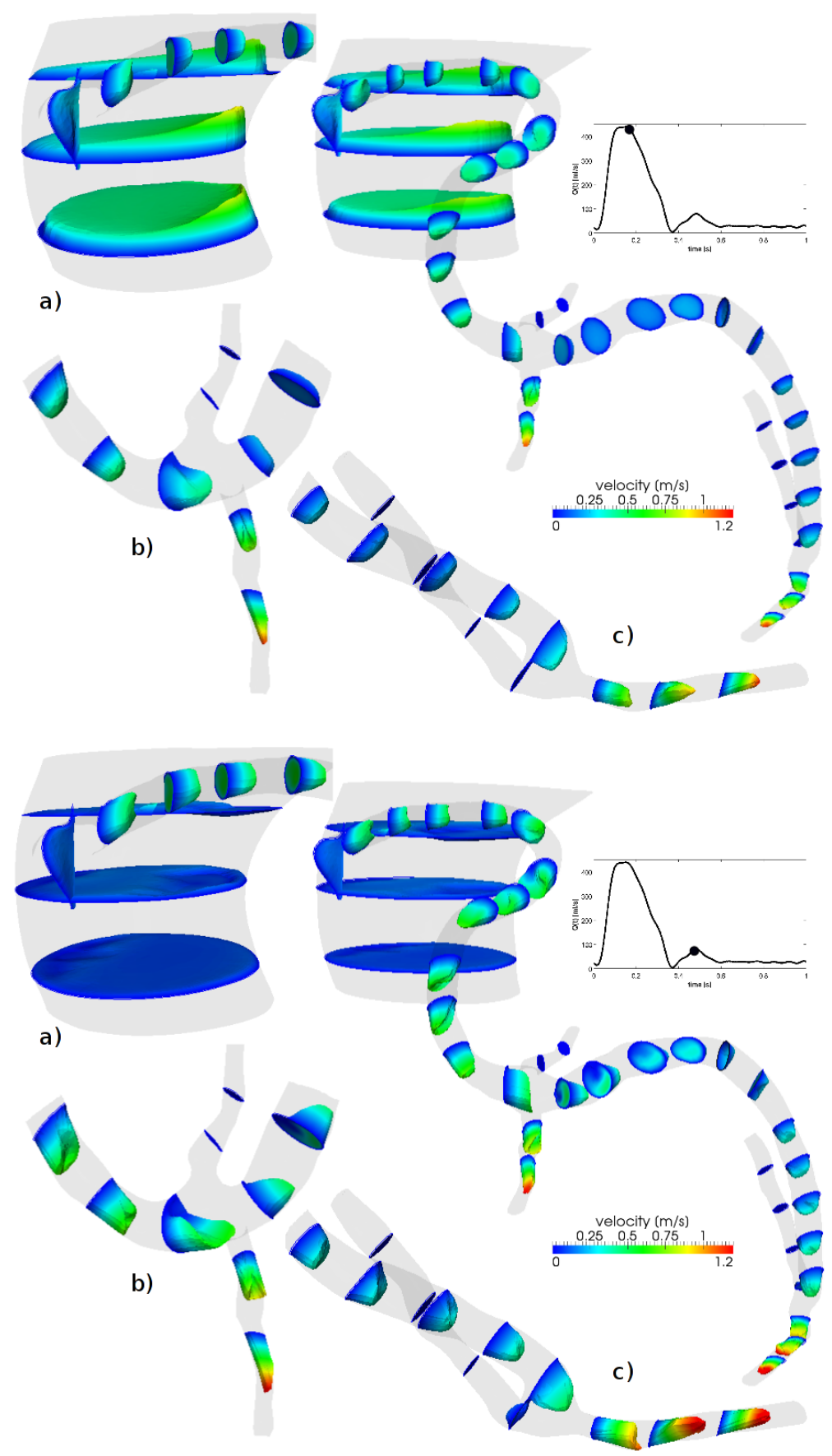

Figure 12. Non-Newtonian blood flow in the double aorto-coronary bypass model - velocity profiles at selected cross-sections at $t_{1}=0.16 \mathrm{~s} \mathrm{(top)} \mathrm{and} t_{2}=0.47 \mathrm{~s}$ (bottom) with detailed views at the (a) proximal anastomosis and (b) distal side-to-side and (c) end-to-side anastomoses. 

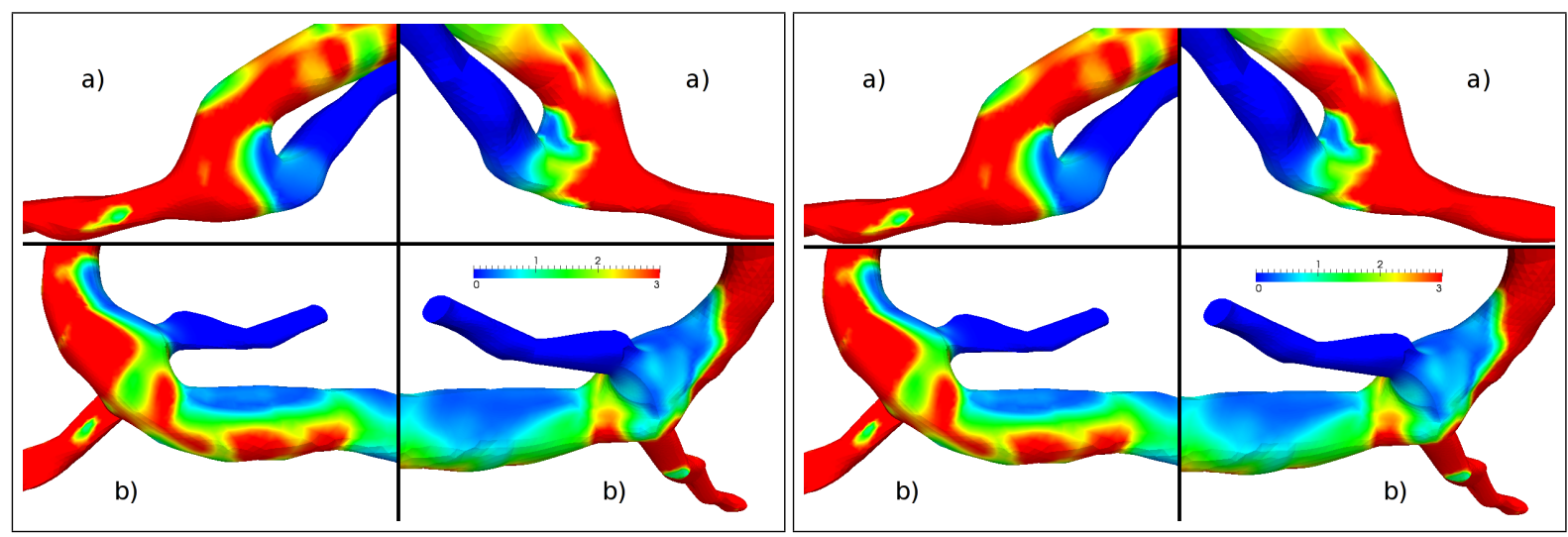

Figure 13. Double aorto-coronary bypass model - distribution of the cycle-averaged WSS magnitude for the Newtonian (left) and non-Newtonian flow (right) with detailed views at the distal (a) end-to-side and (b) side-to-side anastomoses of the sequential graft.
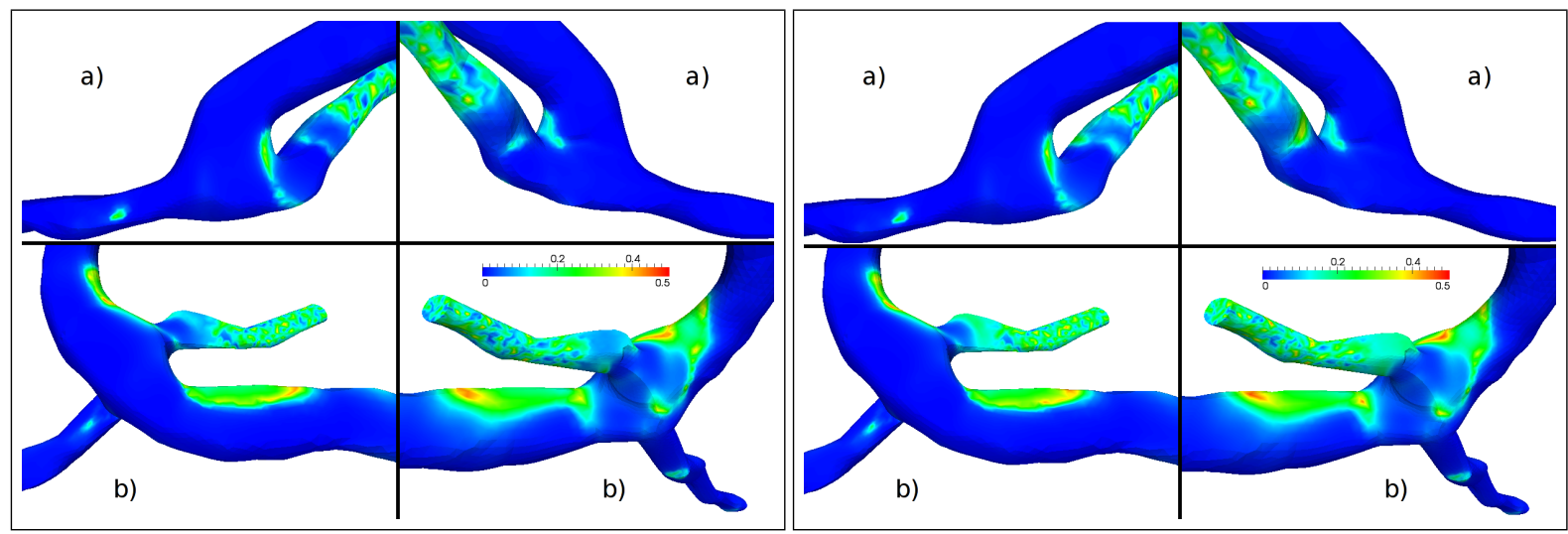

Figure 14. Double aorto-coronary bypass model - distribution of the OSI for the Newtonian (left) and non-Newtonian flow (right) with detailed views at the distal (a) end-to-side and (b) side-to-side anastomoses of the sequential graft.

(right). At this point, it is also interesting to note that the sequential graft maintains higher velocity magnitudes most of the cardiac cycle in comparison to the individual graft. Regarding the values of the cycle-averaged WSS magnitude for the Newtonian and non-Newtonian blood flow, Fig. 16, we come to the same conclusion as we did for the single and double bypass models. Namely, that no significant non-Newtonian effects are observed at the distal anastomoses. This statement is further confirmed with the OSI distribution shown in Fig. 17, where the differences between the Newtonian and non-Newtonian flows are negligible.

\section{CONCLUSION}

Comparing the numerical results for the pulsatile Newtonian and non-Newtonian blood flow in the single, double and triple aorto-coronary bypasses, it was interesting to note that the consideration of blood's non-Newtonian viscosity had almost no effect on the distribution of WSS and OSI. Because both wall parameters are known to be sensitive to any change in the hemodynamics, it is possible to conclude that blood's non-Newtonian behaviour may be neglected in the case of coronary bypasses. This conclusion corresponds to our observations 


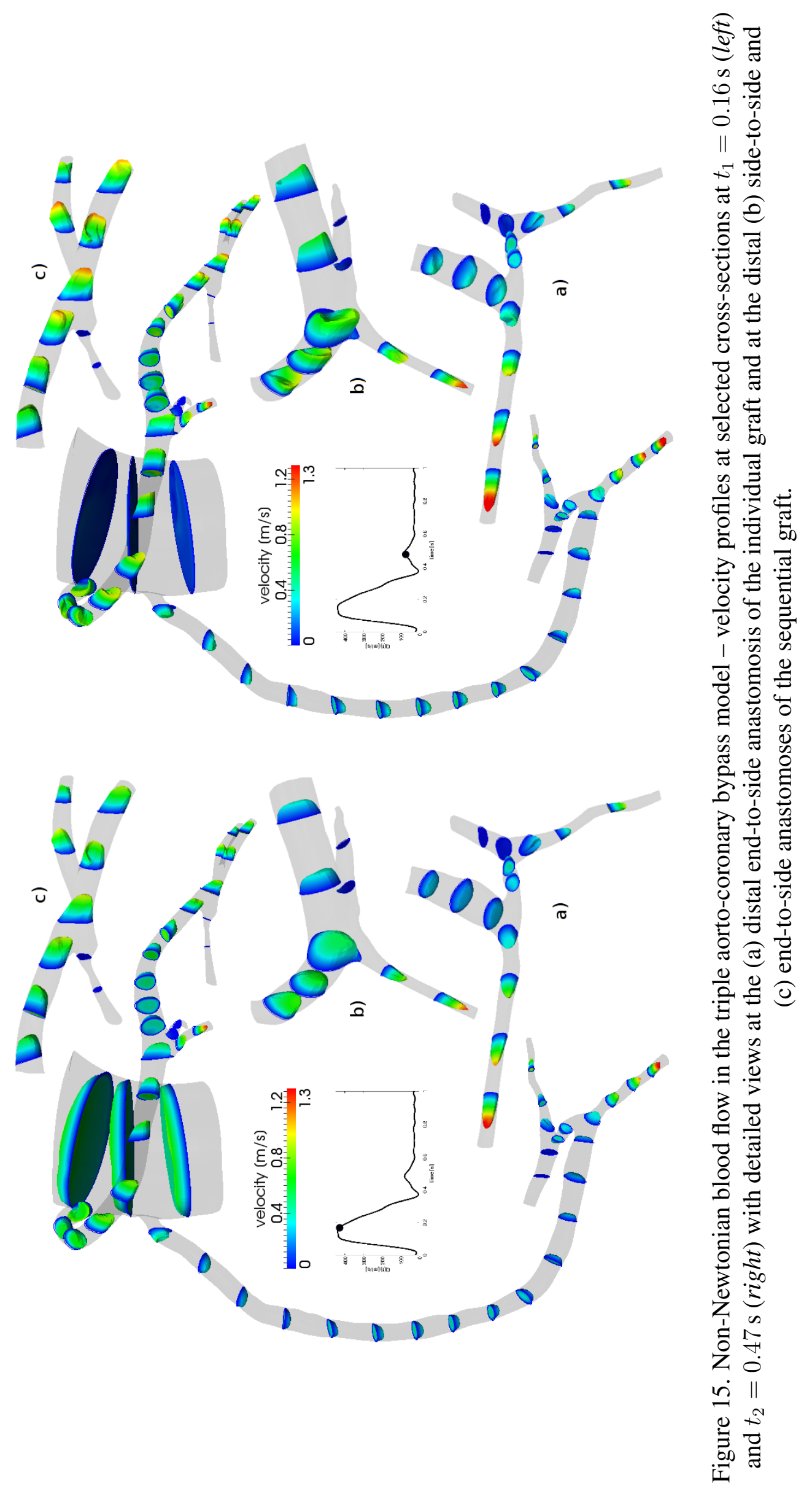



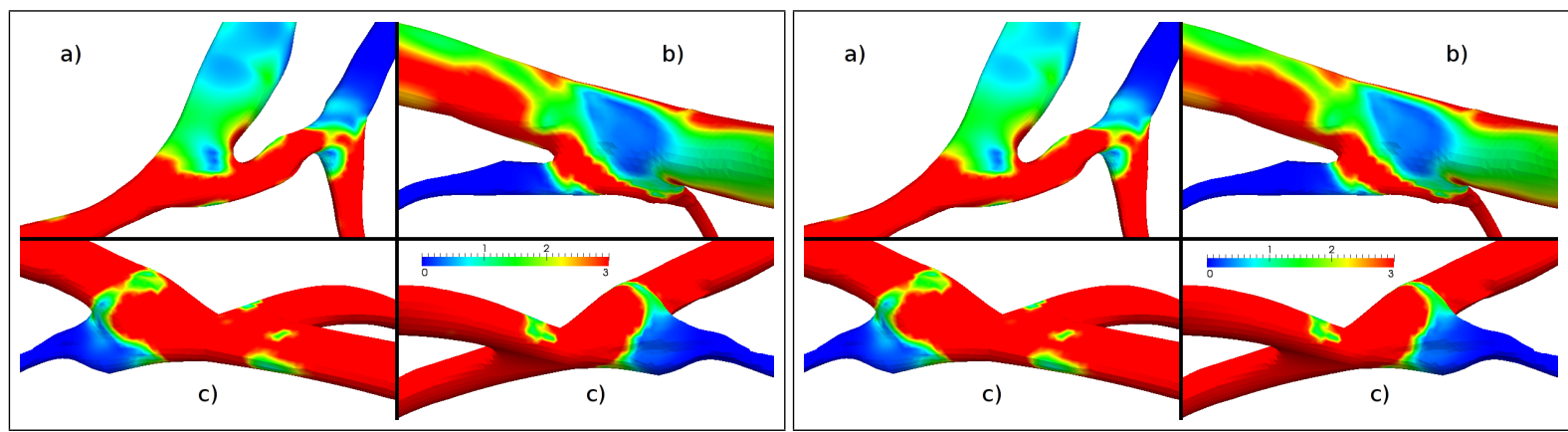

Figure 16. Triple aorto-coronary bypass model - distribution of the cycle-averaged WSS magnitude for the Newtonian (left) and non-Newtonian flow (right) with detailed views at the (a) distal end-to-side anastomosis of the individual graft and at the distal (b) side-to-side and (c) end-to-side anastomoses of the sequential graft.
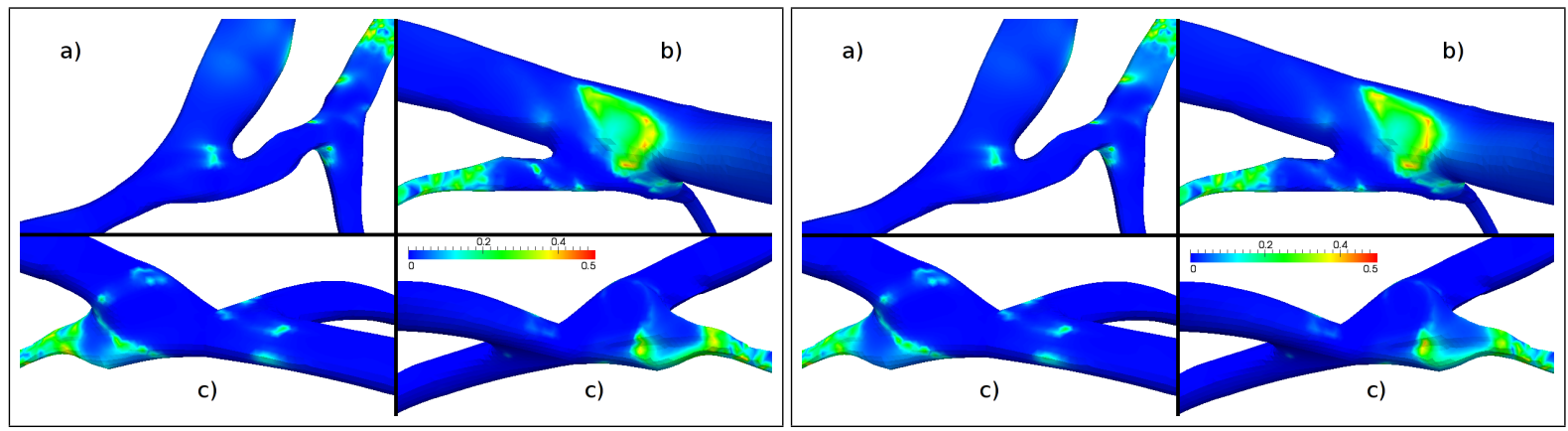

Figure 17. Triple aorto-coronary bypass model - distribution of the OSI for the Newtonian (left) and non-Newtonian flow (right) with detailed views at the (a) distal end-to-side anastomosis of the individual graft and at the distal (b) side-to-side and (c) end-to-side anastomoses of the sequential graft.

made in our previous study [13], where we dealt with steady non-Newtonian blood flow in idealised bypass models.

We are aware that the present study has several limitations. First of them is the assumption of inelastic walls, which especially for the in reality elastic aorta is a major drawback. In the future, we hope to improve the present model by simulating fluid-structure interactions. Another possibility of improvement may be seen in the prescription of physiologically correct boundary values at the coronary outlets.

\section{Acknowledgements}

This study was supported by the European Regional Development Fund (ERDF), project "NTIS - New Technologies for Information Society", European Centre of Excellence, CZ.1.05/1.1.00/02.0090. 


\section{REFERENCES}

[1] Bassiouny H. S., White S., Glagov S., Choi E., Giddens D. P., Zarins C. K., "Anastomotic intimal hyperplasia: Mechanical injury or flow induced". J. Vasc. Surg. 15, 708-717, 1992.

[2] Barth T. J., Jesperson D. C., "The design and application of upwind schemes on unstructured meshes". AIAA Paper 89-0366, 1989.

[3] Cho Y. I., Kensey K. R., "Effects of the non-Newtonian viscosity of blood on flows in diseased arterial vessels. Part I: Steady flows". Biorheology 28, 241-262, 1991.

[4] Ferziger J. H., Perić M. Computational methods for fluid dynamics, Springer, 1999.

[5] Frauenfelder T., Boutsianis E., Schertler T., Husmann L., Leschka S., Poulikakos D., Marincek B., Alkadhi H., "Flow and wall shear stress in end-to-side and side-to-side anastomosis of venous coronary artery bypass grafts". Biomed. Eng. Online 6, 35, 2007.

[6] Haruguchi H., Teraoka S., "Intimal hyperplasia and hemodynamic factors in arterial bypass and arteriovenous grafts: A review". J. Artif. Organs 6, 227-235, 2003.

[7] He X., Ku D. N., "Pulsatile flow in the human left coronary artery bifurcation: Average conditions". J. Biomech. Eng. 118, 74-82, 2012.

[8] Kabinejadian F., Chua L. P., Ghista D. N., Sankaranarayanan M., Tan, Y. S., "A novel coronary artery bypass graft design of sequential anastomoses". Ann. Biomed. Eng. 38, 3135-3150, 2010.

[9] Kim D., Choi H., "A second-order time accurate finite volume method for unsteady incompressible flow on hybrid unstructured grids". J. Comput. Phys. 162, 411-428, 2000.

[10] Leuprecht A., Kozerke S., Boesiger P., Perktold K., "Blood flow in human ascending aorta: A combined MRI and CFD study". J. Eng. Math. 47, 387-404, 2003.

[11] Loth F., Fischer P. F., Bassiouny H. S., "Blood flow in end-to-side anastomoses". Аnnu. Rev. Fluid Mech. 40, 367-393, 2008.

[12] Olufsen M. S., Peskin C. S., Kim W. Y., Pedersen E. M., Nadim A., Larsen J., "Numerical simulation and experimental validation of blood flow in arteries with structured-tree outflow conditions". Ann. Biomed. Eng. 28, 1281-1299, 2000.

[13] Vimmr J., Jonášová A., "Non-Newtonian effects of blood flow in complete coronary and femoral bypasses". Math. Comput. Simulat. 80, 1324-1336, 2010.

[14] Vural K. M., Şener E., Taşdemir O., "Long-term patency of sequential and individual saphenous vein coronary bypass grafts". Eur. J. Cardio-Thorac. 19, 140-144, 2001.

[15] Xiong F. L., Chong C. K., "A parametric numerical investigation on haemodynamics in distal coronary anastomoses". Med. Eng. Phys. 30, 311-320, 2008.

[16] Zeng D., Ding Z., Friedman M. H., Ethier C. R., "Effects of cardiac motion on right coronary artery hemodynamics". Ann. Biomed. Eng. 31, 420-429, 2003.

[17] Zhang J. M., Chua L. P., Ghista D. N., Yu S. C. M., Tan Y. S., "Numerical investigation and identification of susceptible sites of atherosclerotic lesion formation in a complete coronary artery bypass model". Med. Biol. Eng. Comput. 46, 689-699, 2008. 